\title{
Diferencias entre hombres y mujeres mayores en funcionamiento cognitivo y calidad de vida
}

\author{
Elena Navarro ${ }^{1}, \mathrm{M}^{\mathrm{a}}$ Dolores Calero ${ }^{1}$ y $\mathrm{M}^{\mathrm{a}} \mathrm{J}$ osé Calero-García ${ }^{2}$ \\ ${ }^{1}$ Universidad de Granada (España); ${ }^{2}$ Universidad de Jaén (España)
}

\begin{abstract}
En la actualidad, existe aún cierta controversia sobre la influencia de la variable sexo sobre el funcionamiento cognitivo y la calidad de vida en la vejez. El principal objetivo del estudio que se presenta es analizar las posibles diferencias entre hombres y mujeres mayores en relación con sus habilidades cognitivas y su calidad de vida. La muestra del estudio está formada por 264 personas mayores de entre 65 y 95 años de las provincias de Jaén y Granada que fueron evaluadas con una prueba de screening cognitivo, un test de fluidez verbal, una tarea de atención sostenida, una prueba de evaluación del potencial de aprendizaje, una tarea de memoria de trabajo y un cuestionario de calidad de vida. Los resultados muestran que, una vez que se controla el nivel educativo y la edad de los participantes, los hombres muestran un rendimiento superior en tareas de screening cognitivo, memoria de trabajo, atención sostenida y fluidez verbal; mientras que las mujeres rinden por encima de los hombres en tareas de memoria y aprendizaje verbal. En calidad de vida, los hombres muestran una mejor salud y una mayor independencia, mientras que las mujeres puntúan por encima de los hombres en integración social y uso de servicios sociales.
\end{abstract}

Palabras clave: Funcionamiento cognitivo, vejez, calidad de vida, diferencias en función de sexo.

Differences between elderly women and men in cognitive functioning and quality of life. Today, there is still some controversy about the influence of the variable sex on cognitive functioning and quality of life in old age. The main objective of the study presented is to analyze possible differences between older men and women in relation to their cognitive abilities and quality of life. The study sample consists of 264 persons aged between 65 and 95 years from the provinces of Jaen and Granada who were assessed with a cognitive screening test, a verbal fluency test, a task of sustained attention, a task to assess learning potential, a working memory task, and of quality of life questionnaire. The results show that, once the educational level and age of participants is controlled, men show superior performance on cognitive screening tasks, working memory, sustained attention and verbal fluency; while women performance above men in verbal memory tasks and verbal learning. In quality of life, men show better health and greater independence, while women scored higher than men on social integration and use of social services.

Key words: Cognitive functioning, old age, quality of life, differences based on gender.

Correspondencia: Elena Navarro González. Facultad de Psicología. Departamento de Personalidad, Evaluación y Tratamiento Psicológico. Universidad de Granada. Campus de Cartuja, s/n. C.P.: 18071. Granada (España). E-mail: enavarro@ugr.es 
La posible existencia de diferencias en función del sexo en rendimiento cognitivo a lo largo de la vida y en especial en la vejez es un tema que desde hace décadas viene generando numerosas investigaciones y que ha arrojado, como veremos a continuación, resultados contradictorios (Faille, 2006; Kaufman, Kaufman, Liu y Johnson, 2008). Tradicionalmente, se ha afirmado que existe un perfil cognitivo diferenciado entre hombres y mujeres, y se ha pensado que las mujeres son mejores en aquellas habilidades relacionadas con lo verbal, mientras que los hombres serían superiores en habilidades de tipo visoespacial (Kaufman et al., 2008). Algunos estudios sostienen que este perfil cognitivo diferenciado entre hombres y mujeres se da desde edades tempranas (Jorm, Anstey, Christensen y Rodgers, 2004; Strand, Deary y Smith, 2006). Así, Frias, Nilsson y Herlitz (2006) confirmaron la estabilidad de dichas diferencias cuando estudiaron a cinco grupos de edad de personas de entre 35 y 80 años durante un periodo de 10 años y encontraron que las mujeres eran mejores en tareas de memoria episódica, de reconocimiento verbal y de caras y en tareas de fluidez semántica, mientras que los hombres eran mejores en tareas como el subtest de cubos del WAIS (Wechsler, 1981).

Smith y Baltes (1998) señalaron en el "Berling Aging Study (BASE) que en una amplia muestra de hombres y mujeres de entre 75 y 105 años había una proporción significativamente mayor de mujeres con sospecha de demencia, y que además las puntuaciones en el Mini-Mental State Examination (MMSE; Folstein, Folstein y McHugh, 1975) eran significativamente inferiores en las mujeres. Según estos autores, los hombres eran superiores a las mujeres tanto en razonamiento (inteligencia fluida) como en conocimiento general (inteligencia cristalizada), si bien señalaban que probablemente tales diferencias pudieran ser debidas a las diferencias previas en cuanto al nivel educativo. Estos hallazgos han sido corroborados por otras investigaciones posteriores (Java, Sulkava, Verkkoniemi y Niinistö, 2001; Yi, Yuzhi y George, 2003).

No obstante, no todos los estudios apuntan a la existencia de tales diferencias en perfil cognitivo entre hombres y mujeres ancianos. Así, Stein et al. (2011) no encontraron diferencias consistentes y sistemáticas entre hombres y mujeres ni en fluidez verbal, ni en nominación, praxias constructivas o reconocimiento a partir de los 75 años, lo cual podría indicar que las diferencias encontradas en algunos estudios se podrían deber a que se trabaja con muestras de menor edad, unidas a otras variables importantes como el nivel educativo (Stein et al., 2011). En este sentido, debe tenerse en cuenta que los estudios realizados han trabajado normalmente con muestras de personas en distintas etapas de la vida, desde la juventud hasta edades avanzadas (Kaufman et al., 2008; Maitland, Hetlitz, Nyberg, Bäckman y Nilsson, 2004). Esto hace que podamos especular que ciertas variables no controladas sean las responsables de las diferencias encontradas y de los resultados contradictorios encontrados. Entre estas variables, cabe señalar, la selección de las muestras y de los participantes y la mayor presencia de mujeres en 
etapas finales de la vida, lo que evidentemente sesga las diferencias en perfil cognitivo entre hombres y mujeres en la vejez. Otra variable no controlada podría ser el nivel educativo, el cual ha mostrado su papel predictivo en el funcionamiento cognitivo general en diversos estudios (Alley, Suthers y Crimmins, 2007; Andel, Vigen, Mack, Clarck y Gatz, 2006).

En relación con la plasticidad cognitiva en la vejez, Faille (2006) encontró que tanto los hombres ancianos como las mujeres poseían una plasticidad cognitiva similar ya que ambos grupos se beneficiaban en igual medida del entrenamiento en memoria. Este resultado es consistente con las conclusiones realizadas por Hyde (2005), quien plantea la hipótesis de la similitud "inter-sexos" para explicar, la ausencia de diferencias significativas en la plasticidad cognitiva en la vejez.

Otro ámbito en el que se ha estudiado si existen o no diferencias en la vejez en función del sexo ha sido en la calidad de vida. La Organización Mundial de la Salud define la calidad de vida como " la percepción de una persona sobre su situación vital, dentro del contexto cultural en el que vive, en relación con sus objetivos, sus expectativas, sus valores e intereses" (WHO, 1993, p. 223). En este sentido, debemos señalar que en la vejez, tanto hombres como mujeres deben enfrentarse a experiencias que disminuyen su calidad de vida como son el deterioro, la dependencia, la soledad, el aislamiento social, etc. (Camargos, Machado y Rodrigues, 2007; Zunzunegui, Alvarado, Beland y Vissandjee, 2009). Algunas investigaciones apuntan que las mujeres podrían tener peor calidad de vida que los hombres en la senectud (Camargos et al., 2008) y de hecho, según un estudio reciente realizado por Drumond, Egüez, Lebrao, de Oliveira y Ferreira (2011), el 28\% de las mujeres a partir de los 60 años de edad tienen dificultades en al menos una actividad básica para la vida diaria, y aunque viven más tiempo, lo hacen a costa de un menor nivel de autonomía y de más necesidades asistenciales en la vejez (Camargos et al., 2007, 2008; Palloni, McEniry, Wong y Peláez, 2006; Zunzunegui et al., 2009).

De la revisión realizada se desprende que si bien la literatura apunta hacia la existencia de una mejor calidad de vida de los hombres en la vejez, en el caso del funcionamiento cognitivo las diferencias no son tan claras siendo la edad una de las variables implicadas en los resultados contradictorios encontrados. Por este motivo se ha considerado relevante en este estudio analizar las diferencias en funcionamiento cognitivo y en calidad de vida entre hombres y mujeres mayores controlando las variables edad y nivel educativo de los participantes. 


\section{MÉTODO}

\section{Participantes}

Un total de 264 ancianos han participado en este estudio. La edad media ha sido de 79,57 años (d.t.=7,7, rango de edad=65-96). El porcentaje de hombres ha sido del $36,7 \%$ de la muestra $(n=97)$ y el de mujeres del 63,3\% (n=167). En esta muestra, el $84 \%$ no tenían estudios formales, el 9,5\% tenían educación primaria y el 6,5\% tenían educación secundaria o superior.

\section{Instrumentos}

Mini-Examen Cognoscitivo (MEC) (Lobo, Ezquerra, Gómez, Sala y Seva, 1979). Esta versión es la traducción y la adaptación española del Mini-Mental State Examination (MMSE, Folstein et al., 1975). El MEC es un instrumento de screening ampliamente utilizado en la detección del deterioro cognitivo (Diniz, Yassuda, Nunes, Radanovic y Forlenza, 2007) que explora de forma rápida y estandarizada un conjunto de funciones cognitivas (orientación temporo-espacial, memoria inmediata y a largo plazo, atención, cálculo, lenguaje, razonamiento abstracto y praxias) que pueden estar afectadas en personas mayores. La puntuación final obtenida en esta prueba se utiliza habitualmente como índice global y como método de seguimiento evolutivo de las funciones cognitivas en procesos como el deterioro cognitivo y la demencia (Calero, Navarro, Robles y García-Berbén, 2000).

Auditory Verbal Learning Test-Potencial de Aprendizaje (AVLT-PA: adaptación española de Calero, 2001; Calero y Navarro, 2006). Se trata de una versión de la prueba tradicional de evaluación de la memoria verbal de Rey (Rey, 1964). En ella, se presentan quince palabras que el sujeto evaluado debe repetir inmediatamente después de haberlas escuchado. En la versión de potencial de aprendizaje, la lista de palabras se presenta seis veces. Las dos primeras repeticiones de la lista hacen de pretest (presentación estándar), las dos siguientes son de entrenamiento (incluyen retroalimentación sobre la ejecución, refuerzo y repetición de palabras no recordadas) y finalmente, las dos últimas repeticiones hacen de postest (presentación estándar y sin retroalimentación). Las puntuaciones con las que se ha trabajado en este estudio han sido: (1) AVLT-PA pre: la puntuación media obtenida en los dos primeros ensayos; (2) AVLP-PA post: puntuación media en los dos últimos ensayos; (3) AVLT-PA puntuación de ganancia: la diferencia entre el AVLT-PA pre y el AVLT-PA post; y AVLT-PA Recuerdo demorado (Rd): número de palabras de la lista recordadas después de pasar una tarea de interferencia. Estudios previos han demostrado que el entrenamiento que se da en la fase intermedia produce mejoras significativas en el rendimiento de los evaluados. La puntuación de ganancia se ha establecido como una 
medida de plasticidad cognitiva en diferentes poblaciones como personas con demencia o esquizofrenia (Wiedl, Schöttke y Calero, 2001).

Tarea de fluidez verbal categorial (Spreen y Strauss, 1998). En ella se les solicita a los participantes que durante un minuto refieran tantos palabras (en este caso, animales) como les sea posible. La fluidez verbal mide principalmente la velocidad y facilidad de producción verbal; además, evalúa la disponibilidad para iniciar una conducta en respuesta ante una tarea novedosa (Lezak, 1995). Asimismo, valora las funciones del lenguaje (denominación, tamaño del vocabulario), la velocidad de respuesta, la organización mental, las estrategias de búsqueda, así como la memoria a corto y largo plazo (Ruff, Light, Parker y Levin, 1997).

Tarea de Atención Sostenida (TAS, Calero y Salguero, 2009). Se trata de una tarea computarizada que consiste en la aparición aleatoria en intervalos de un segundo de números del 1 al 9, debiendo pulsar la barra espaciadora inmediatamente después a la aparición de la secuencia "3-6". Existe un bloque inicial de ensayos de entrenamiento y cuatro bloques posteriores de prueba. Cada bloque experimental tiene una duración de 1 minuto y en cada uno aparece aleatoriamente la secuencia "3-6" quince veces sobre 60 secuencias posibles, siendo las 45 secuencias restantes consideradas como distractores. La variable dependiente en esta tarea es el número de aciertos totales por participante.

Tarea de Memoria de Trabajo (TM, Oakhill, Yuill y Parkin, 1989). Esta tarea mide la amplitud de la memoria de trabajo a partir de la presentación de tarjetas con tres números cada una, de manera que la persona debe leer en voz alta los números de cada tarjeta y recordar el último número que se le presenta, ya que luego tendrá que reproducirlos una vez que se le hayan presentado todas las tarjetas de cada uno de los bloques. El número de tarjetas se va incrementando en función del rendimiento de la persona, de manera que en el primer bloque, la tarea empieza con dos tarjetas, mientras que en el último bloque se presentan cinco tarjetas.

Cubrecavi (Cuestionario Breve de Calidad de Vida de Fernández-Ballesteros y Zamarrón, 2007). Es un cuestionario que fue diseñado para la evaluación multidimensional de la calidad de vida en la vejez. Con esta prueba se obtiene información sobre: salud en general (subescalas "salud objetiva" y "salud subjetiva"), integración social del sujeto, habilidad para el funcionamiento cotidiano y la independencia, nivel de actividad y participación en actividades de ocio y tiempo libre, la percepción subjetiva sobre la calidad del entorno en que vive el anciano, satisfacción con la vida, educación recibida, estimación de los ingresos económicos del individuo y finalmente el uso que hace el anciano de los recursos socio-sanitarios disponibles. Este cuestionario ha sido validado tanto en población española como en población suramericana (Fernández-Ballesteros y Zamarrón, 2007), mostrando una buena consistencia interna (entre 0.70 y 0.92 ). 


\section{Procedimiento, diseño y análisis estadístico}

Para la selección de los participantes de las residencias se pidió al equipo de las mismas que hicieran una preselección de participantes con las siguientes características: edad igual o superior a 65 años, ausencia de enfermedad grave o demencia (criterios DSM-IV-TR); y ausencia de déficits motores o sensoriales. Para los participantes que vivían en sus casas, las personas fueron seleccionadas de entre aquellas que acudían a la Universidad de Mayores de la Universidad de Granada o de Centros de Día una vez que a partir de una entrevista inicial cumplían con los criterios de inclusión antes citados. A cada participante seleccionado se le explicó de manera individual el objetivo del estudio para que dieran el consentimiento informado si querían participar en el mismo. Las evaluaciones las realizaron psicólogos experimentados en dos sesiones con una duración máxima de una hora. En la primera sesión se pasaba una entrevista semi-estructurada, el CUBRECAVI y el MEC. En una segunda evaluación se pasaban el resto de las pruebas. El estudio siguió un diseño cuasi-experimental ex post facto. Teniendo en cuenta los objetivos, la muestra se clasificó en función de la variable sexo estableciéndose dos grupos: 97 hombres y 167 mujeres.

Para analizar las diferencias entre grupos en funcionamiento cognitivo y calidad de vida, se llevó a cabo un modelo linear general multivariante en el que se incluyó el nivel educativo (años de educación) y la edad como covariables. Las variables dependientes fueron las descritas en el apartado de "instrumentos". La variable independiente fue el sexo de los participantes. Los datos fueron analizados con el paquete estadístico SSPS 15.0.

\section{RESULTADOS}

El objetivo de este estudio fue analizar el posible efecto del variable sexo en el funcionamiento cognitivo y en la calidad de vida de los participantes. Puesto que en análisis estadísticos preliminares se vio que el nivel educativo estaba positivamente relacionado con el rendimiento en todas las pruebas cognitivas utilizadas y la variable edad, correlacionaba negativamente y de manera significativa $(p<0.01)$ con todas ellas, para analizar el funcionamiento cognitivo se realizó un Modelo lineal general controlando estadísticamente el nivel educativo y la edad como covariables (ver tabla 1). Tal y como se muestra en la tabla, los resultados indican que -una vez controladas ambas variables- existe un efecto significativo a favor de los hombres en el MEC $\left(F_{(3,194)}=18.412, p=0.0001\right)$, en la FVS $\left(F_{(3,194)}=13.660, p=0.0001\right)$, en la TAS $\left(F_{(3,194)}=15.3534, p=0.0001\right)$ y en la tarea de memoria de trabajo $\left(F_{(3,194)}=24.878\right.$, $p=0.0001$ ), mientras que las mujeres muestran un rendimiento significativamente superior en el AVLT-PA pre $\left(F_{(3,194)}=19.023, p=0.0001\right)$, en el AVLT-PA post $\left(F_{(3,194)}=16.717, p=0.0001\right)$, en el AVLT-PA-Rd $\left(F_{(3,194)}=15.108, p=0.0001\right)$ y en la 
NAVARRO et al. Diferencias entre hombre y mujeres en rendimiento y calidad de vida en la vejez.

puntuación de ganancia en el AVLT-PA $\left(F_{(3,194)}=5.821, p=0.001\right)$. No obstante, el tamaño del efecto en todos los casos fue pequeño $\left(\eta^{2}<0.28\right)$.

Tabla 1. Efecto de la variable sexo sobre las distintas variables cognitivas contempladas en el estudio, controlando la edad y el nivel educativo como covariables

\begin{tabular}{|c|c|c|c|c|c|c|c|}
\hline \multirow{3}{*}{ Variables cognitivas } & \multicolumn{4}{|c|}{ Sexo } & \multirow{3}{*}{$F_{(3,194)}$} & \multirow{3}{*}{$\eta^{2}$ parcial } & \multirow{3}{*}{ Potencia observada } \\
\hline & \multicolumn{2}{|c|}{ Hombres } & \multicolumn{2}{|c|}{ Mujeres } & & & \\
\hline & Media & d.t. & Media & d.t. & & & \\
\hline MEC & 23.195 & 4.443 & 21.845 & 5.4328 & $18.412 * *$ & 0.222 & 1.000 \\
\hline FVS & 12.134 & 4.808 & 11.024 & 4.2638 & $13.660^{* *}$ & 0.174 & 1.000 \\
\hline Aciertos TAS & 38.732 & 16.88 & 36.425 & 15.353 & $15.279 * *$ & 0.191 & 1.000 \\
\hline AVLT-PA pre & 3.895 & 1.722 & 4.164 & 1.7036 & $19.023 * *$ & 0.227 & 1.000 \\
\hline AVLT-PA post & 7.574 & 3.290 & 8.534 & 3.3216 & $16.717 * *$ & 0.205 & 1.000 \\
\hline AVLT-PA-Rd & 6.151 & 3.848 & 7.126 & 4.0011 & $15.108 * *$ & 0.189 & 1.000 \\
\hline PG. AVLT-PA & 3.592 & 2.251 & 3.955 & 2.3425 & $5.821 * *$ & 0.083 & 0.949 \\
\hline M. trabajo & 2.005 & 1.241 & 1.771 & 1.1427 & $24.878 * *$ & 0.278 & 1.000 \\
\hline
\end{tabular}

Nota: MEC: Mini-Examen Cognoscitivo; FVS: fluidez verbal semántica; Aciertos TAS: aciertos en la tarea de atención sostenida; AVLT-PA pre: puntuación pre en el test de aprendizaje auditivo-verbal, versión potencial de aprendizaje: AVLT-PA post: puntuación post en el test de aprendizaje auditivo-verbal versión potencial de aprendizaje; AVLT-PA-Rd: puntuación en la variable recuerdo demorado del test de aprendizaje auditivo-verbal, versión potencial de aprendizaje; PG. AVLT-PA: puntuación ganancia en el test de aprendizaje auditivo verbal, versión potencial de aprendizaje; M. trabajo: rendimiento en la tarea de memoria de trabajo. $* * p<0.01$

En segundo lugar se analizó la influencia de la variable sexo en la calidad de vida de los ancianos. Se realizó un Modelo lineal general multivariado para analizar las posibles diferencias existentes entre hombres y mujeres en las distintas dimensiones que componen el Cubrecavi controlando igualmente el nivel educativo y la edad de los participantes. Al analizar los efectos principales, los resultados mostraron la existencia de diferencias estadísticamente significativas a favor de los hombre en salud objetiva

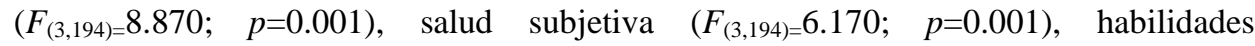

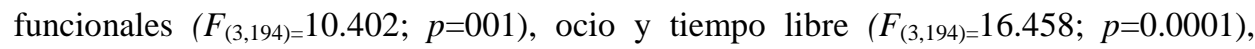
mientras que las mujeres obtienen puntuaciones significativamente superiores en integración social $\left(F_{(3,194)=} 6.451 ; p=0.001\right)$, y uso de servicios sociales $\left(F_{(3,194)=8.870 \text {; }}\right.$ $p=0.001)$. De nuevo, en todos los casos, el tamaño del efecto fue bajo.

Tabla 2. Influencia de la variable sexo sobre la calidad de vida de los ancianos, controlando el nivel educativo y el grupo de edad de los participantes como covariables

\begin{tabular}{|c|c|c|c|c|c|c|c|}
\hline \multirow{3}{*}{ Dimensiones Cubrecavi } & \multicolumn{4}{|c|}{ Sexo } & \multirow{3}{*}{$F_{(3,260)}$} & \multirow{3}{*}{$\eta^{2}$ parcial } & \multirow{3}{*}{ Potencia observada } \\
\hline & \multicolumn{2}{|c|}{ Hombres } & \multicolumn{2}{|c|}{ Mujeres } & & & \\
\hline & Media & d.t. & Media & d.t. & & & \\
\hline Salud objetiva & 3.3493 & 0.38828 & 3.2149 & 0.43812 & $8.870 * *$ & 0.093 & 0.995 \\
\hline Salud subjetiva & 2.7749 & 0.91788 & 2.6948 & 0.86655 & $6.170 * *$ & 0.066 & 0.961 \\
\hline Salud mental & 3.0590 & 0.66420 & 2.9069 & 0.64076 & 2.355 & 0.026 & 0.587 \\
\hline Habilidades funcionales & 3.0601 & 0.89599 & 2.8467 & 0.91802 & $10.402 * *$ & 0.107 & 0.999 \\
\hline Ocio/tiempo libre & 2.1318 & 0.53235 & 2.1136 & 0.53233 & $16.458 * *$ & 0.160 & 1.000 \\
\hline Integración social & 2.1181 & 0.49426 & 2.1893 & 0.52245 & $6.451 * *$ & 0.069 & 0.968 \\
\hline Satisfacción vital & 2.6722 & 0.91705 & 2.7976 & 0.83420 & 1.625 & 0.018 & 0.424 \\
\hline Uso SS.SS. & 2.8804 & 0.63322 & 2.9560 & 0.56221 & $3.608 *$ & 0.040 & 0.790 \\
\hline
\end{tabular}

Nota: Actividades OTL: actividades de ocio y tiempo libre; SS.SS.: uso de los servicios sociales y sanitarios.

$* p<0.05 ; * * p<0.01$ 


\section{DISCUSIÓN}

Como se comentó anteriormente, nuestro principal objetivo fue determinar si existen o no diferencias en funcionamiento cognitivo y calidad de vida en mujeres y hombres mayores.

Los resultados obtenidos, nos muestran que -una vez que se controla la variable edad y nivel educativo- las mujeres tienen un mejor rendimiento en la tarea de memoria verbal tanto en el recuerdo a corto plazo como en el recuerdo a largo plazo de la lista de palabras. Igualmente, también obtienen una puntuación superior en plasticidad cognitiva evaluada a partir de la puntuación de ganancia en la prueba AVLT-PA. Estos resultados confirman la hipótesis de que las mujeres en la vejez muestran un rendimiento superior a los hombres en tareas de memoria de tipo verbal (Aarsten, Martin y Zimprich, 2004; Herlitz y Rehnman, 2008). No obstante, estudios previos hacían prever un mejor rendimiento en fluidez verbal en las mujeres (Gerstorf et al., 2006). Sin embargo, nuestro estudio no encontró tal diferencia, obteniendo los hombres un rendimiento superior en esta tarea. En este aspecto, se corrobora el estudio reciente de Stein et al. (2011) que tampoco encontró diferencias entre hombres y mujeres en tareas de fluidez verbal. Según este autor, el sexo de los participantes es menos determinante que otras variables socio-demográficas a la hora de determinar el funcionamiento cognitivo en la vejez. De hecho, el nivel educativo en nuestro estudio explica casi el 45,8\% de la variabilidad de los resultados obtenidos con una potencia observada de 1 , muy superior al $16,5 \%$ de la variabilidad explicada por la edad o al $17,9 \%$ explicada por la variable sexo de los participantes.

Resulta también interesante que las mujeres presentan una mayor capacidad de aprendizaje verbal, como se refleja en el hecho de que presentan mayores puntuaciones de ganancia en el AVLT-PA, índice considerado como indicativo de plasticidad cognitiva en la vejez (Calero y Navarro, 2007). En el resto de las pruebas, los hombres muestran un rendimiento significativamente superior, hecho que se observa en el MEC, en la prueba de fluidez, en la tarea de atención sostenida y en memoria de trabajo. Estos resultados confirman los resultados obtenidos por Smith y Baltes (1998) en uno de los primeros estudios en los que se analizó las diferencias en funcionamiento cognitivo entre hombres y mujeres en la vejez. Así, la presente investigación confirma la existencia de un perfil cognitivo diferenciado entre hombres y mujeres en la vejez y al mismo tiempo demuestra la importancia de controlar tanto el nivel educativo como la edad de los ancianos cuando se intenta determinar la posible existencia de diferencias en funcionamiento cognitivo en diferentes áreas o en plasticidad cognitiva.

En relación con la calidad de vida, diversos estudios previos sugerían que los hombres tenían una calidad de vida en la vejez superior a la de las mujeres (Camargos et al., 2007; Drumond et al., 2011). Sin embargo, nuestros resultados mostraron la 
existencia de diferencias entre hombres y mujeres en determinadas escalas de las evaluadas por el CUBRECAVI, de tal manera que los hombres obtenían puntuaciones indicativas de una mayor calidad de vida en salud subjetiva y objetiva, y en habilidades funcionales, al igual que mostraron menos quejas sobre distintos síntomas físicos y una mayor independencia que las mujeres. Estos resultados concuerdan con estudios previos, donde los ancianos varones parecen tener menores problemas físicos a edades avanzadas (Lym, Paik y Park, 2008). No obstante, las mujeres puntuaron significativamente por encima de los hombres en m integración social y en uso de servicios sociales, variables que podrían relacionarse con la búsqueda de ayudas para superar sus problemas físicos.

A pesar de los datos mostrados anteriormente, estos resultados deberían ser replicados con muestras más amplias de ancianos que incluyeran poblaciones de otras regiones y con niveles educativos altos. Finalmente, sería interesante controlar otras variables como el nivel económico de la muestra por su posible influencia específicamente sobre la calidad de vida, hecho que no pudimos hacer en este estudio por respeto a la confidencialidad de los participantes en la investigación.

\section{REFERENCIAS}

Aartsen, M.J., Martin, M. y Zimprich, D. (2004). Gender differences in level and changes in cognitive functioning: results from the Longitudinal aging study Amsterdam. Gerontology, 50(1), 35-38.

Alley, D., Suthers, K. y Crimmins, E. (2007). Education and cognitive decline in older Americans: Results from de AHEAD simple. Research on Aging, 29, 73-94.

Andel, R., Vigen, C., Mack, W.J., Clark, L.J. y Gatz, M. (2006). The effect of educational and occupational complexity on rate of cognitive decline in Alzheimer patients. Journal of the International Neuropsychological Society, 12, 147-52.

Calero, M.D., Navarro, E., Robles, P. y Berbén, T.M. (2000). Estudio de validez del Mini-examen Cognoscitivo de Lobo y cols. para la detección del deterioro cognitivo asociado a demencias. Neurología, 15(8), 337-354.

Calero, M.D. y Navarro, E. (2007). Cognitive plasticity as a modulating variable on the effects of memory training in elderly persons. Archives of Clinical Neuropsychology, 22, 63-72.

Calero, M.D. y Salgado A. (2009). TAS: Tarea de atención sostenida. Granada. Ed. Sinder

Camargos, M.C., Machado, C.J. y Rodrigues, R.N. (2007). Disability life expectancy for the elderly, city of São Paulo, Brazil, 2000: Gender and educational differences. Journal of Biosocial Science, 39, 455-463.

Camargos, M.C., Machado, C.J. y Rodrigues, R.N. (2008). Life expectancy among elderly Brazilians in 2003 according to different levels of functional disability. Cadernos de Saúde Pública/Ministério da Saúde, Fundação Oswaldo Cruz, Escola Nacional de Saúde Pública, 24, 845-852.

Deary, I.A., Whiteman, M.C., Starr, J.M., Whalley, L.J. y Fox, H.C. (2004). The impact of childhood intelligence on later life: Following up the Scottish Mental Survey of 1932 and 1947. Journal of Personality and Social Psychology, 86, 130-147.

Diniz, B., Yassuda, M., Nunes, P., Radanovic, M. y Forlenza, O. (2007). Mini Mental-State Examination performance in mild cognitive impairment subtypes. 
Drumond, F., Guevara, P., Lebrao, M., Oliveira, Y. y Ferreira, J. (2011). Gender Differences in Life Expectancy and Disability-Free Life Expectancy Among Older. Women's Health Issues, 21, 64-70.

Faille, L. (2006). Performance on a brain-plasticity-based memory training computer program for the elderly as influenced by cognitive functioning and gender. Department of Psychology, California School of Professional Psychology, San Francisco, EEUU.

Fernández-Ballesteros, R., Zamarrón, M.D. y Maciá, A. (2000). Calidad de vida en la vejez en distintos contextos. 1st ed. Madrid: INSERSO.

Fernández-Ballesteros, R. y Zamarrón, M.D. (2007). Cubrecavi. Cuestionario breve de calidad de vida. Madrid: TEA Ediciones.

Folstein, M.E, Folstein, S.E. y McHugh, P.R. (1975). "Mini Mental State": A practical method for grading the cognitive state of patients for the clinician. Journal of Psychiatric Research, 12, 189-198.

Gerstorf, D., Herlitz, A. y Smith, J. (2006). Stability of sex differences in cognition in advanced old age: the role of education and attrition. Journal of Gerontology, 61(4), 245-249.

Hale, S., Rose, N.S., Myerson, J., Strube, M.J., Sommers, M., Tye-Murray, N. y Spehar, B. (2011). The structure of working memory abilities across the adult life span. Psychology and Aging, 26, 92-110. doi:10.1037/a0021483

Herlitz, A. y Rehnman, J. (2008). Sex differences in episodic memory. Current Directions in Psychological Science, 17(1), 52-56.

Hyde, J.S. (2005). The gender similarities hypothesis. The American Psychologist, 60(6), 581-592.

Java, K., Sulkava, R., Verkkoniemi, A. y Niinistö, L. (2001). Sex differences in cognitive performance among the very old Mini-Mental State Examination in a population aged 85 years and over. Journal of Clinical Neuropsychology, 7(1), 39-45.

Jorm, A.F., Anstey, K.J., Christensen, H. y Rodgers, B. (2004). Gender differences in cognitive abilities: The mediating role of health state and health habits. Intelligence, 32, 7-23.

Kaufman, A.S., Kaufman, J.C., Liu, W. y Johnson, C.K. (2008). How do educational attainment and gender relate to fluid intelligence, crystallized intelligence, and academic skills at ages 22-90 years? Archives of Clinical Neuropsychology, 24, 153-163.

Lezak, M.D. (1995). Neuropsychological assessment. New York: Oxford University Press.

Lobo, A., Ezquerra, J., Gómez, F., Sala, J. y Seva, A. (1979). El Mini-Examen-Cognoscitivo. Un test sencillo y práctico para detectar alteraciones intelectuales en pacientes médicos. Actas Luso Españolas de Neurología y Psiquiatría, 19(7), 189-201.

Lym, J., Paik, N. y Park, W. (2008). The effect of age and sex on physical function and quality of life in Korean elderly: The Korean Longitudinal Study on Health and Aging. Archives of Physical Medicine and Rehabilitation, 89, E52.

Maitland, S.B., Hetlitz, A., Nyberg, L., Bäckman, L. y Nilsson, L. (2004). Selective sex differences in declarative memory. Memory and Cognition, 32(7), 1160-1169.

Oakhill, J., Yuill, N. y Parkin, A. (1989). Working memory, comprehension ability and the resolution of text anomaly. British Journal of Psychology, 80, 351-61.

Ruff, R.M., Ligth, R.H., Parker, S.B. y Levin, H.S. (1997). The psychological construct of word fluency. Brain and Language, 57, 349-405.

Smith, L. y Baltes, M. (1998). The role of gender in very old age: profiles of functioning and everyday life patterns. Psychology and Aging, 13(4), 676-695.

Spreen, O. y Strauss, E. (1998). A compendium of neuropsychological tests: Administration, norms, and commentary. New York: Oxford University Press.

Stein, J., Luppa, M., Luck, T., Maier, W., Wagner, M., Daerr, M., van den Bussche, M., et al. (2011). The Assessment of changes in cognitive functioning: age, education, and genderspecific reliable change indices for older adults tested on the CERAD-NP battery: 
Results of the German Study on Aging, Cognition, and Dementia in primary care patients (AgeCoDe). American Journal of Geriatric Psychiatry, 19(2), 1-14.

Weschler, D. (1981). Manual for the Wechsler Adult Intelligence Scale-Revised. New York: The Psychological Corporation.

World Health Organization (WHO). Group Study protocol fr the WHO project to develop a quality of life assessment instrument (WHOQoL). Quality of Life Research, 2, 153-159.

Wiedl, K.H., Schöttke, H. y Calero, M.D. (2001). Dynamic assessment of cognitive rehabilitation potential in schizophrenic persons and in old people with and without dementia. International Journal of Psychological Assessment, 17, 112-119.

Yi, Z., Yuzhi, L. y George, L. (2003). Gender differentials of the oldest old in China. Research on Aging, 25(1), 65-80.

Zunzunegui, M.V., Alvarado, B.E., Beland, F. y Vissandjee, B. (2009). Explaining health differences between men and women in later life: A cross-city comparison in Latin America and the Caribbean. Social Science and Medicine, 68, 235-242.

Recibido: 30 de abril de 2014 Recepción Modificaciones: 26 de mayo de 2014

Aceptado: 7 de agosto de 2014 